\title{
Astrometry for New Reductions: The ANR method
}

\author{
Vincent Robert ${ }^{1,2}$ and Christophe Le Poncin-Lafitte ${ }^{3}$ \\ ${ }^{1}$ IMCCE, Observatoire de Paris, PSL Research University, CNRS, Sorbonne Universités, \\ UPMC Univ. Paris 06, Univ. Lille 1, \\ 77 avenue Denfert-Rochereau, F-75014, Paris, France \\ email: vincent.robert@obspm.fr \\ ${ }^{2}$ IPSA, Institut Polytechnique des Sciences Avancées, \\ 63 bis boulevard de Brandebourg, F-94200, Ivry-sur-Seine, France \\ ${ }^{3}$ SYRTE, Observatoire de Paris, PSL Research University, CNRS, Sorbonne Universités, \\ UPMC Univ. Paris 06, LNE, \\ 61 avenue de l'Observatoire, F-75014, Paris, France \\ email: christophe.leponcin@obspm.fr
}

\begin{abstract}
Accurate positional measurements of planets and satellites are used to improve our knowledge of their orbits and dynamics, and to infer the accuracy of the planet and satellite ephemerides. With the arrival of the Gaia-DR1 reference star catalog and its complete release afterward, the methods for ground-based astrometry become outdated in terms of their formal accuracy compared to the catalog's which is used. Systematic and zonal errors of the reference stars are eliminated, and the astrometric process now dominates in the error budget.

We present a set of algorithms for computing the apparent directions of planets, satellites and stars on any date to micro-arcsecond precision. The expressions are consistent with the ICRS reference system, and define the transformation between theoretical reference data, and groundbased astrometric observables.
\end{abstract}

Keywords. Astrometry, Catalogs, Methods: miscellaneous

\section{Introduction}

Accurate orbit determination requires a large amount of observations over a large time span to allow for the best precision and extrapolation. Such kind of observations were processed a long time ago with inaccurate star catalogs and methods, compared to recent ones. No real efforts have been attempted to reanalyze these data a new time, considering the amount of time, mean and energy required. Using photographic plates of planetary satellites, we demonstrated that a new reduction of old observations can improve significantly the ephemerides (Arlot et al. 2012, Robert et al. 2011, Robert et al. 2015, Robert et al. 2016, Lainey et al. 2017). More important, we showed that ephemerides can sometimes be significantly biased while their extrapolation quickly diverges, due to several reasons and in particular the imprecision of past observations that are introduced in the adjustments.

In this framework and with support of the Gaia mission, the NAROO project has been initiated at Paris Observatory with the primary aim to reprocess the old astrometric observations with the best instrumental, algorithmic and numerical techniques. We attempt to answer the following question: how to ensure the Gaia accuracy over one century? 


\section{The astrometry in the Gaia era}

The "classical" astrometric method to compute high-precise apparent places of the objects consists in correcting reference positions for all known effects (Kaplan et al. 1989). In particular the proper motions with first-order models, the parallaxes with geometric transformations, the aberrations and light deflection with geometric transformations and relativistic terms, and the atmospheric refraction with semi-analytical solutions. The key point is that the "classical" accuracy of the overall astrometric process is about 20 mas, that is to say $60 \mathrm{~km}$ at the distance of Jupiter, or $120 \mathrm{~km}$ at the distance of Saturn.

The example of the proper motions. 1st-order models of propagation are enough accurate since the star catalog errors are dominating and $\dot{r}$ is not known:

$$
\left\{\begin{aligned}
\Delta \alpha \cos \delta & =\mu_{\alpha}\left(t-t_{0}\right) \\
\Delta \delta & =\mu_{\delta}\left(t-t_{0}\right)
\end{aligned}\right.
$$

But since the Gaia catalog errors are up to 1000 times more accurate than other astrometric references, one should use 3rd-order models of propagation to ensure the catalog accuracy:

$$
\left\{\begin{aligned}
\Delta \alpha \cos \delta & =\mu_{\alpha}\left(t-t_{0}\right)-\left(\dot{r} \mu_{\alpha}-\tan \delta_{0} \mu_{\alpha} \mu_{\delta}\right)\left(t-t_{0}\right)^{2}+\xi_{\alpha}\left(t-t_{0}\right)^{3} \\
\Delta \delta & =\mu_{\delta}\left(t-t_{0}\right)-\left(\dot{r} \mu_{\delta}+\frac{1}{2} \tan \delta_{0} \mu_{\alpha}^{2}\right)\left(t-t_{0}\right)^{2}+\xi_{\delta}\left(t-t_{0}\right)^{3}
\end{aligned}\right.
$$

The example of the calibration models. N-order conventional calibration models are used to map the transformation between the measured $(x ; y)$ and tangential $(X ; Y)$ frames. But since the Gaia external accuracy is up to 1000 times more accurate than other astrometric references, one should refine the developments at least with 1st-order coma and magnitude, 2nd-order tilt, 3rd-order distorsion, and temperature terms to traduce and measure all physical effects that could not be analyzed before:

$$
\left\{\begin{array}{l}
X=\rho_{x} \cos \theta_{x} x-\rho_{y} \varphi(T) \sin \theta_{x} y+\Delta_{x}+C_{x} x \Delta m+p x^{2}+q x y+D x\left(x^{2}+y^{2}\right) \\
Y=\rho_{x} \sin \theta_{y} x+\rho_{y} \varphi(T) \cos \theta_{y} y+\Delta_{y}+C_{y} y \Delta m+p x y+q y^{2}+D y\left(x^{2}+y^{2}\right)
\end{array}\right.
$$

\section{The ANR project}

Our work aims at providing complete set of algorithms to compute the apparent place of the objects in the sky, observed from ground. While the most accurate star catalogs provided an overall accuracy about of 1 mas, the Gaia catalog improved the positioning accuracy with that of $1 \mu$ as. In the same way and while the method of Kaplan et al. (1989) provided an overall accuracy about of 1 mas, we aim for that of $1 \mu$ as for ground observations.

\section{References}

Arlot, J.-E., Desmars, J., Lainey, V., \& Robert, V. 2012, PSS, 73, 1

Kaplan, G. H., Hughes, J. A., Seidelmann, P. K., Smith, C. A., \& Yallop, B. D. 1989, AJ, 97

Lainey, V., Jacobson, R. A., Tajeddine, R., Cooper, N. J., Murray, C., Robert, V., Tobie, G., Guillot, T., Mathis, S., Remus, F., Desmars, J., Arlot, J.-E., De Cuyper, J.-P., Dehant, V., Pascu, D., Thuillot, W., Le Poncin-Lafitte, C., \& Zahn, J.-P. 2017, Icarus, 281

Robert, V., de Cuyper, J.-P., Arlot, J.-E., de Decker, G., Guibert, J., Lainey, V., Pascu, D., Winter, L., \& Zacharias, N. 2011, MNRAS, 415, 1

Robert, V., Lainey, V., Pascu, D., Pasewaldt, A., Arlot, J.-E., De Cuyper, J.-P., Dehant, V., \& Thuillot, W. 2015, A\&SA, 582, A36

Robert, V., Pascu, D., Lainey, V., Arlot, J.-E., De Cuyper, J.-P., Dehant, V., \& Thuillot, W. 2016, $A \mathscr{E} A, 596, \mathrm{~A} 37$ 